\title{
Crossmedia Systems Constructed around Human Activities: A Field Study and Implications for Design
}

\author{
Katarina Segerståhl \\ University of Oulu, Department of Information Processing Science \\ P.O. Box 3000, FIN-90014 Oulu, Finland \\ katarina.segerstahleoulu.fi
}

\begin{abstract}
Many interactive systems today span across a range of interoperable IT artifacts, forming crossmedia systems. They aim at providing pervasive and synergistic support for human activities. This paper reports a three-month-long qualitative field study exploring the use of a crossmedia fitness system to support physical training. The main concern is how the system - through the configuration of its components - supports the primary activity. Users' primary motivation, elaborateness of their activities, internalization or externalization of their actions and their perceived threshold toward using distinct IT artifacts determined the utilization of the system and each of its components. Compositional aspects of the system, such as its hierarchical structure, distribution of functionality and functional modularity influenced its ability to support different ways of training. The article contributes by shedding light on aspects that influence the synergistic use of IT artifacts and by proposing implications for designing crossmedia systems.
\end{abstract}

Keywords: Pervasive computing, crossmedia systems, interaction design, human activity, case study.

\section{Introduction}

The purpose of this study is to understand (and ultimately to help design for) a complex interaction - such as where multiple devices and applications are used together to carry out a set of interrelated tasks. The focus is on crossmedia systems, i.e., combinations of interoperable media (devices and applications) that are systemically constructed around a distinct space or activity.

Recent emphasis in mobile computing on 'smart' and one-size-fits-all design has led to the "chaos of infinite combinatorial possibilities" [10]. Yet, there exists a manageable range of situated types or recognizable spaces of human activities, e.g., learning, collaborating, shopping, and sporting [10]. Design that is targeted on a few appropriate themes is more likely to be appreciated within those spaces [10]. Alongside the development of all-power-in-one devices, there appears to be growing interest in developing crossmedia systems that are purposefully structured around a specific space. Examples include: a system for realtors to manage open houses and sales [1]; laboratory information management systems [13]; the Nike + iPod, and the Polar training systems for fitness and sports; as well as systems for managing personal 
information across mobile and non-mobile use contexts (e.g., smartphones that connect with calendars and other applications on laptops and desktop computers).

What is common for all of these systems is that they aim at providing pervasive task support through an optimal configuration of multiple intertwined IT artifacts that each address distinct contextual settings of the primary activity. Devices and applications in such configurations are often retrospectively bridged. This may be the case when existing technologies are combined to form mash-ups, applications are built on legacy platforms, or when the components of a system have been designed in different organizations or teams. Technical interconnectivity is often achieved, but actual interoperability (in terms of affordance for combinatorial use) has not always been supported all the way. Also, the situated and subjective nature of human activity is challenging to design for. However, crossmedia systems hold potential for supporting a range of human activities that could be better utilized by focusing on their structure and composition.

This study investigates the use of a system that is designed to support physical training. As is characteristic of crossmedia, the system is designed with distinct presuppositions about how the training activity is structured. I will investigate, how the system composition, i.e., the roles and functionality of its components and the ways in which they are intended to work together, actually corresponds with users' real-world training practices. Based on my observations, I will discuss implications for the design of crossmedia systems in terms of their structure and composition.

In the next section I will briefly introduce the background for the study; then I will describe the field study as well as its findings and analysis. In the final sections I will discuss the implications of the study focusing on the design of crossmedia systems.

\section{Background}

In 2000 Steven Feiner [5] introduced the concept of hybrid user interfaces in which multiple heterogeneous displays and interaction devices are used in "synergistic combination to benefit from the advantages of each" [5]. One example of this vision in practice is the iRoom which was created in an exploratory project at Stanford University. The iRoom is an interactive workspace where multiple technologies are mapped to a single defined physical location and configured to provide combinatorial support for meeting activities [7]. Today, there are a number of information systems that expand across diverse applications and devices. Yet, despite technical advances and increased interoperability, there are still issues that remain to be addressed regarding the everyday interactions with these systems. This is especially the case when crossmedia systems expand across both stationary and mobile use contexts.

Oulasvirta's and Sumari's [14] research outlines several strategies that users develop and adopt for putting together sets of media to support their tasks. Users employ these behavioral strategies in order to get the best out of the information appliances that they carry around with them, at the same time trying to fit them into their environments [14]. Users constantly find new ways of coping with and using information technology by carrying out the practice of recombinant computing, i.e., serendipitously interweaving diverse media to enrich information and to gain pervasive 
task support $[12,14]$. Feiner predicted that such use of hybrid user interfaces is difficult to predict and structure, and may become so diversified that special 'environment management' will be needed [5].

However, the systems could as well be designed focusing on pre-identified situated spaces. This would provide grounds for incorporating environment management into the systems' composition. Applying the concept of crossmedia to the design of information systems may help to support the different aspects of human activities and form a conceptual basis for designing systems that may better afford the synergistic use of their components.

\subsection{Crossmedia Systems and Synergistic Specificity}

The term crossmedia has emerged in the context of modern communications research converging with the fields of pervasive computing and human-computer interaction (HCI) [20]. According to Filgueiras et al. [6], crossmedia can be defined as "the collaborative support of multiple media to delivering a single story or theme, in which the storyline directs the receiver from one medium to the next, according to each medium's strength to the dialogue." Crossmedia may not only deliver a story or theme, but may also be used for supporting a specific goal or objective through functionality that is distributed across media [3]. According to Boumans [3] the characteristics of crossmedia include that, more than one medium is involved in supporting a message/story/goal, and that the delivery or support of the common message/story/goal purposefully spans across the different media.

What distinguishes crossmedia systems from, for example cross-platform systems is that instead of enabling an application to run on multiple platforms or for content to be accessible through multiple media, it is spread across them for optimization of modalities and interaction resources. Crossmedia systems reach their full intended potential when their components are used in combination, or synergistically. Schilling [16] has termed synergistic specificity as "the degree to which a system achieves greater functionality by its components being specific to one another" within a particular configuration. Systems high in synergistic specificity may be able to provide functionality that more modular systems cannot.

The functionality of some systems relies on optimizing the components' ability to work with each other. In such systems, detaching the components or using them in isolation would result to a loss in performance or even paralyze the whole system [18]. An example of high synergistic specificity can be found in the Apple iPod products where the mobile devices are dependent on the desktop application as the source of power and content. There are also crossmedia systems that are more flexible due to a higher degree of modularity, i.e., they can be used in different combinations and parts of them can be operated independently. A more modular system achieves synergy when used in such a way that the benefits of its components are merged.

Crossmedia systems are designed by establishing interoperable combinations of media around a distinct theme (space or activity) and developed with the intent of enabling and supporting the synergistic use of their components. In the next section I will report the findings of a field study that explores the use of a crossmedia system in real-world settings. 


\section{Field Study: Training with the Polar Fitness System}

The focus of our investigation is in how a crossmedia system, through the configuration of its components, supports people in training to maintain and improve their physical fitness. The study was carried out in cooperation with Polar Electro, a world leading manufacturer of heart rate monitoring and sports technology [9]. The Polar fitness system, comprising a wearable heart rate monitor and an interoperable web service was used as an example of a crossmedia system.

\subsection{The Polar Fitness System}

The Polar fitness system is targeted to active, fitness-oriented, but ordinary users thus excluding, e.g., professional athletes. It comprises a wearable heart rate monitor, the Polar FT60, and a web service, www.polarpersonaltrainer.com. Along with the heart rate monitor, accessories such as a GPS receiver, a heart rate monitoring strap, and a USB dock for transferring data to the web are included.

The wrist unit provides a fitness program with weekly targets that help in managing exercising throughout the week. It also provides information and instruction during exercise and helps the user to achieve desired heart rate zones and other exercise related goals. After each exercise the wrist unit provides feedback and a weekly summary with suggestions for the upcoming week. The web service includes a training calendar and tools for creating long-term training programs and detailed exercise plans, and information and instructions for heart-rate-based exercise. It also provides progress charts, graphs and summaries for analytic and long-term follow up, a place to document exercising and a long-term storage for exercise data. Figure 1 illustrates how the system and its components are intended to support the different aspects of the training activity.

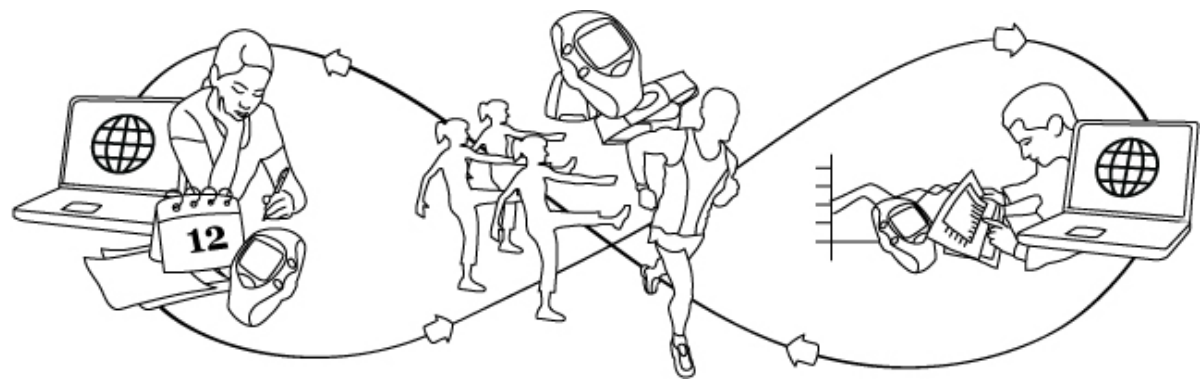

Fig. 1. The Polar fitness system spans across the training activity via its components by providing support for planning, exercise and follow-up

\subsection{Method and Approach}

A qualitative multiple case field study was conducted with 12 participants over a period of three months. The aim was to investigate and understand the aspects in users' training practices that influenced the use of the fitness system and each of its components. Through this understanding it was possible to analyze how the system structure corresponded with the actual training activity. 
The study was announced at two fitness centers and participants could apply by filling in an application. A total of 30 applications were received out of which 12 applicants were selected to take part in the study. The method of purposeful intensity sampling [15] was used for selecting the participants. The sample was constructed to match the criteria of potential real-world users that had previously been identified by Polar. Within these criteria, a sample that expressed diversity in terms of age, gender, and professional background as well as activity and training background was formed.

Four group interviews were carried out throughout the study, each followed by a three-week-long self-documentation period when the participants used the target technology in their daily lives. Participants filled out cross-sectional questionnaires at each group meeting. During the self-documentation periods, structured diaries were filled in by participants on a daily basis. During the study each participant was observed and interviewed. Observations were one and a half hours each and they were conducted applying the principles of contextual inquiry [2]. The inquiries were carried out either at a gym when the participant was performing a regular workout or by joining the participant's run or jog. Research instruments such as diaries and questionnaires were developed upon analysis throughout the study. See Fig. 2 for an overview of the data collection process. All inquiries were recorded and documented with notes. Interviews were recorded and professionally transcribed and diary and questionnaire input were extracted to tables for analysis. The grounded theory [17] approach was applied in systematic coding of the data and in discovering emergent categories.

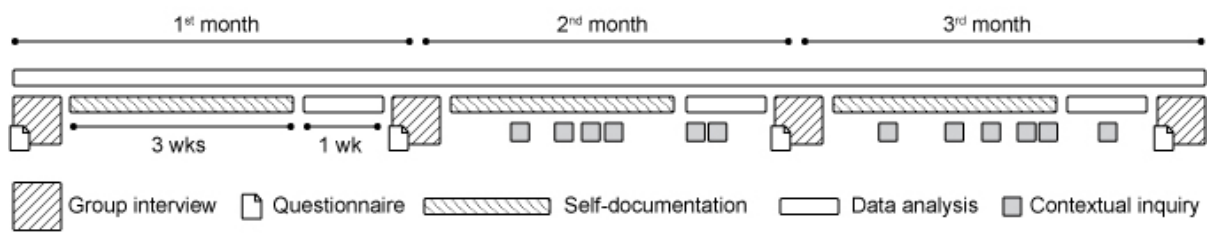

Fig. 2. The data collection process

\subsection{Participants}

Participants were screened according to the pre-identified characteristics of potential real-world users. They were between ages 23 and 40. Seven of them were female and five were male. All participants were active in sports and reported training approximately three to five times a week with a history of regular exercise.

The participants' exercising activities included jogging, cycling, and indoor exercise such as gym workouts or taking group exercise classes. Participants came from various professional backgrounds. Within this group of subjects, it was found that basic skills regarding everyday IT (e.g. PC/laptop, mobile phone, digital camera) use were well established. Experience using internet for daily or utilitarian purposes (browsing, e-mail, internet banking) was also generally common. However experience of combinatory use of multiple devices was not common. The participants and their utilization of the target technology will be investigated in more detail in the following sections. 


\section{Findings}

I will begin by describing the participants and the reasons they had for training. Then I will describe the ways in which they carried out their training related activities. After this I will tackle the use of the fitness system and its components. Finally, I will discuss how the use of target technology and the ways of training fluctuated over the course of the study.

\subsection{Objectives of Training}

As the participants' primary motivation for training and the ways in which they carried out their planning, exercise and follow-up activities were analyzed, three categories of motivational types were identified that corresponded with styles of training. These are improvement $(\mathrm{n}=4)$, self-monitoring $(\mathrm{n}=3)$ and maintenance $(\mathrm{n}=5)$ oriented individuals.

The improvement oriented valued objectives such as performance, fitness, health and enjoyment generally higher than appearance and weight. They also more often set long-term goals for their training, such as an event or a specific improvement in their performance. As one of them told about her goal:

P\#09: “I'm training for my first marathon. To train and improve technique, I'm attending a running school. I also train with my personal trainer once every week. I plan my other exercises so that they support my running practice. "

The self-monitoring subjects considered all objectives, performance, fitness, health and enjoyment, as well as appearance and weight as equally important. However, instead of looking to achieve a specific improvement or goal, they were more curious about how their training generally influenced their personal state regarding each of the objectives. Self-monitoring subjects would enjoy collecting and analyzing data about themselves and their actions even if there was no specific goal or event that they would do it for.

The maintenance-oriented subjects were very much aware of the importance of training and they felt the objectives as the end toward which they should train regularly. As opposed to the improvement and self-monitoring oriented, they would not, however, take on an analytic approach to their training. They had more often difficulties in maintaining a regular exercise regimen and in staying motivated.

The differences identified in the analysis of training objectives were reflected in subjects' individual training styles and became meaningful in the ways in which they would utilize the target technology. The following section will explore the different styles of training that were identified.

\subsection{Styles of Training}

The improvement oriented subjects carried out their planning, exercise and follow-up activities in relatively elaborate ways. They would systematically plan their training several weeks or months in advance. Their exercises were often goal-oriented, including detailed interval-exercises for improving performance as well as lower intensity 
ones to train endurance. They analyzed their performance and progress with calendars, diaries and notebooks usually in relation to their long-term goal.

The self-monitoring oriented subjects were also prone to elaborateness through their curiosity and eagerness to constantly learn more about themselves and their training. Most of them would plan ahead one week at a time (as opposed to training serendipitously or in longer cycles). They would set weekly goals often with respect to an abstract idea of what should be accomplished in terms of diversity and intensity of exercise. When exercising, they often conformed to moderately structured exercises (e.g., warm-up/work/cool-down). The self-monitoring subjects carried out analysis of exercise amounts and intensities with respect to their weekly targets. They would often revisit their exercise data for motivation.

The maintenance oriented subjects, instead of planning or analyzing, would more often exercise on an ad hoc basis. They would not usually plan ahead, but fit exercise wherever they could. Sometimes they would mark down their exercises into calendars and, for motivational purposes, look back at what has been accomplished.

The identified styles of training were not constant. Some fluctuation was observed over the course of the study among all participants. The training activity was fairly sensitive to participants' various life situations, e.g., periodic changes in their work schedule, holidays, and situations in which they needed to prioritize time for family and friends. This became apparent in the participants' diaries:

P\#12: “A couple of busy weeks ahead, going out of town for three days for business and got a couple of exams coming up. So for the next two weeks I will train whenever I can find time. Then I'll hopefully get back into my normal rhythm."

\subsection{Use of Target Technology}

Over the first three weeks of the study the subjects were very enthusiastic about the target technology, namely at this point the heart rate monitor. Their threshold to appropriate it and learn its use was relatively low. After three weeks had passed, participants were explicitly encouraged to explore the web service. This was necessary in order to collect data on the potential uses of the different components. As the users finally started to explore the web service and interoperate the two media, the dynamics behind the process begun to show. Fig. 3 illustrates some of the individual differences in the use of the system that were identified.

The maintenance oriented would use the heart rate monitor for motivation and guidance, but did not see much use in the web service. Four of them had little experience of using the web or any other technologies for anything but mandatory purposes (such as banking) and were reluctant to invest time into learning the use of the web service. They also did not believe that it would provide additional value.

One of them tried it out and was at first very interested, but after a week found that it just wasn't useful enough for her as she only trained a week at a time and for that the heart rate monitor was adequate.

A similar case occurred among the self-monitoring type, where a participant reported being interested in the web service when first exploring it, but abandoned it later because it was too advanced or complicated. She continued to use her traditional calendar and diary for follow up. 


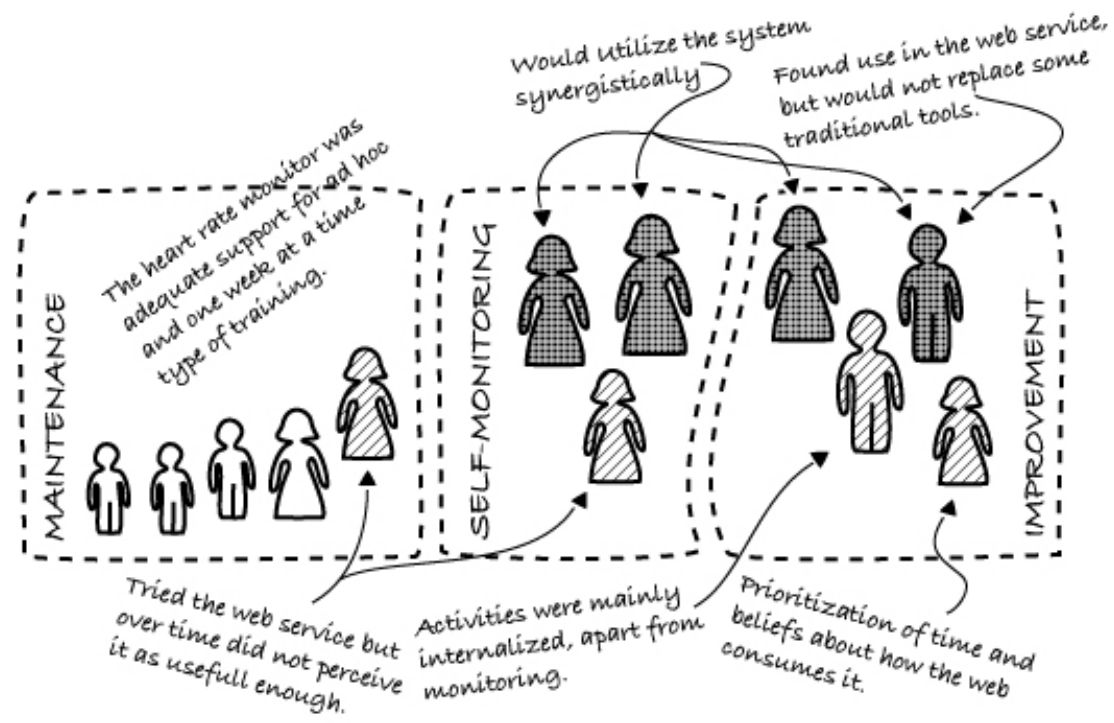

Fig. 3. Summary of individual differences in how participants used the system. Individuals' size indicates the relative elaborateness of their activities and their position indicates their proneness towards utilizing the web service as an addition to the heart rate monitor.

P\#05: "I uploaded my exercises online earlier and it was pretty interesting. But there's no way I can find time for really using it [the web service]. The wrist unit is great, because it's always with me when I exercise and it still stores all my weekly exercises. Right now I just don't need anything more detailed for my purposes."

The self-monitoring and the improvement oriented participants that carried out their exercise at a higher level of elaborateness were generally more interested in the web service. They would carry out their activities at a level where actions more often needed to be externalized with tools, such as notebooks, graphs, and training diaries. For example, one of the participants was interested in running a marathon and felt that she needed all the support to accomplish her training as efficiently as possible. For this purpose, she perceived the web service as useful.

P\#09: “For the past two years I've kept a diary where I've been writing down almost everything about my training. It's so much more convenient to be able to transfer all that information automatically to the computer and it's also easier to analyze it there."

Half of the improvement oriented users would find the web service useful for them, especially in that it provided long-term training programs. They would use these to refresh their exercise routines and to make them more efficient.

There were two participants among the improvement oriented who were less prone towards the web service. One of them had most of his training internalized, i.e., he could memorize the weights he was working out with and had his entire exercise plan 
'in his head'. He also had a clear perception of his longer term goals and was able to follow them as he trained. He had years of experience from team sports, which probably influenced his ability to carry out systematic training with few external tools. He used the heart rate monitor mainly to regulate intensities during exercise and to collect data. The other participant was in a life situation where she had very little time to begin with and perceived the web as being a "time-thief." She was very reluctant to use the web service for her training and felt that it would compromise the little time that she had with family and for actual exercising. She managed her personal goals and improvement with the heart rate monitor and used its fitness-trend feature and weekly summaries to make judgments about her progress.

\section{Analysis and Discussion}

The findings of the study were organized and analyzed using some concepts from activity theory. Activity theory $[8,11]$ is one of the "contextualist" or "postcognitivist" theories used in current HCI as a framework for understanding and analyzing the role and use of modern technology in mediating human activity.

Activity theory is constructed around five key principles, which include 1) intentionality, proposing that an activity is always targeted at an object, i.e., the motivation of the 'doing'; 2) hierarchical structure of activity, suggesting that depending on the level of analysis, an activity can be broken down into temporally and hierarchically related components; sub-activities, actions and operations; 3) internalization/ externalization, meaning that actions may be internalized, i.e., become unconscious or automatic like riding a bicycle, or as actions become more complex, they may be externalized with tools, e.g., using a spreadsheet to carry out complex calculations; 4) tool mediation, emphasizing that both physical as well as conceptual tools or artifacts are a fundamental part of any human activity and play a distinct role in shaping it; and finally 5) historicity/development, stressing that past events contribute to tools' current use and shape. In this study I focus on the mediated relationship on an individual level. As the underlying motivation is in advancing the design of crossmedia systems, I also put special emphasis on the utilization of the target technology across the different cases of training. Using constructs from activity theory as tools, I will first summarize the dynamics of utilization that were observed in this study. Then I will analyze, how real-world practice influenced the use of technology and then, based on these analyses, I will draw implications for how crossmedia systems could better respond to human activity.

\subsection{Dynamics of Utilization}

The participants' primary motivation together with the level of elaborateness that they trained on, were the primary determinants for their proneness towards adopting and using multiple media. The need for external tools was also determined by the level of internalization or externalization of the users' actions. Individual, experiential and social issues as well as the users' life situations also influenced the participants' utilization threshold (or proneness) towards the target technology and its components. (See Fig. 4) 


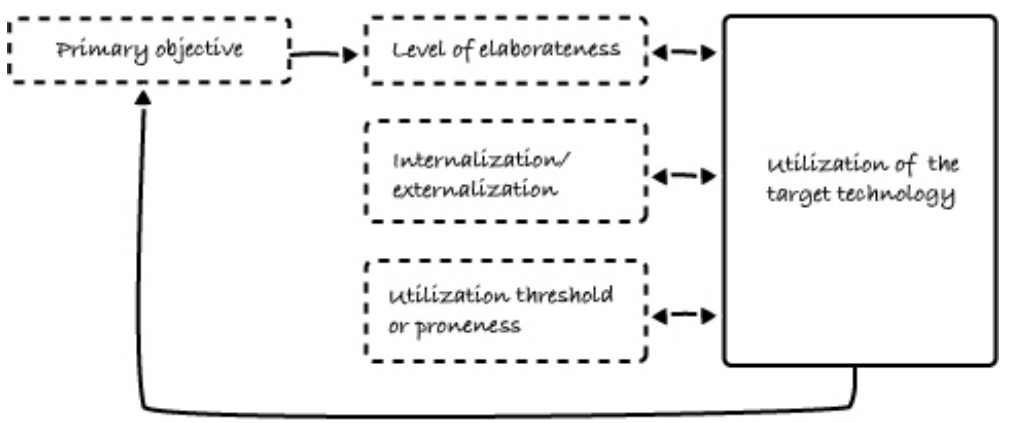

Fig. 4. The dynamics of utilization

As it was used, the fitness system also changed the ways in which subjects trained and sometimes even their primary objectives. For example, one of the participants discovered how he could use heart rate information to regulate his recovery times between weight lifting sets when he was working out at the gym. Another participant would train regularly to maintain his health at the beginning of the study, but set new, more improvement oriented goals for himself as the tools, i.e., the expert training programs, became available to him.

By breaking down the dynamics of utilization and understanding the role of the target technology and its components in each case, it is possible to identify distinct ways in which crossmedia systems could better respond to human activities.

\subsection{Activities in Practice}

In addition to identifying the dynamics of utilization, it is important to understand how they are actuated and shaped in practice. When participants' training was studied in practice, it was found that there were a number of other personal activities, such as work and socializing, that penetrated into the area of the supposed ideal training activity and sometimes distorted it. People's activities are not such isolated ideals, and therefore these ideals cannot function as the sole basis of system design.

The underlying ideal of a distinct situated type of the training activity, fundamentally characterized the composition of the fitness system. One example of this is the assumption that there is such a thing as systematic training that would require more advanced tools (such as the web service) for support. It was assumed that users who would train in this way would find the functionalities as they needed them. However, this need only became apparent when the participants were encouraged to explore the web service - the ideal of the activity did not exist in practice as strong enough to trigger proactive exploration for additional support. Apparently the conceptual representation of a human activity behind the system is not enough - it needs to actively support the synergistic use of its components and support users in finding the functionalities that they may benefit from.

The idea of a distinct situated type or activity is to some degree a functional one when designing crossmedia systems. It is the first step in defining the main task domains that a system should span as well as its basic requirements. However, compositional aspects that allow for crossmedia systems to respond to the fluctuating nature of human activity also need to be taken into account. 


\subsection{Implications for Composition of Crossmedia Systems}

Based on my understanding of how a crossmedia system - through the configuration of its components - may support the different ways of carrying out an activity as well as respond to its individual fluctuations, I will now draw implications for design.

Hierarchical Structure. Each of the devices and applications within a crossmedia system has a distinct role, i.e., each addresses a specific domain of the activity as their primary. For example, the role of the heart rate monitor was to support the exercise context, whereas the role of the web service was to support the planning and analyzing contexts. The roles of the components should not only be considered with respect to the primary activity but also with respect to each other. Users did not always conform to the ideal structure of the training activity. For example, depending on their life situation, they would sometimes not plan their exercising at all and leave followup for later. Exercise, however, was the core activity that could be carried out independently of the other, supporting activities. For exercising, users relied on a single component, the heart rate monitor, as their primary and incorporated the secondary component, the web service, when they needed or had time for it. Thus, the heart rate monitor was the primary component in the system as the source of content (exercise data) and core functionality. Incorporating a hierarchy of components into the system's configuration may increase the system's ability to respond to different user needs and fluctuation of practices.

Distribution of Functionality. The very idea of crossmedia systems is to optimize interaction resources for the different contextual settings that may occur throughout a human activity. When functionalities are appropriately distributed or specialized across the different media within the system they will each contribute to the synergy with their individual strengths. The complexity of a system's individual components as well as redundancy of functionality and content may be reduced by distributing functionality according to the system's hierarchical structure. This means that more advanced functionality, that is likely to benefit a portion of users, could be assigned to an additional media, not necessarily the primary one. Users, who need extended support for their activities, as was observed, may more often have a lower threshold for adopting multiple media. However, with some this threshold is still very high. Users who need the more advanced functionalities, need to be better supported in extending their use practices across more of the systems' components for holistic support. In contrast, users who do not need all the possible functionalities would not be burdened with their added complexity on a single medium.

Functional Modularity. The roles that are assigned to each component help to identify their primary strengths and functions in the system, however, these roles and strengths should not be too strictly defined. As was found, people may carry out an activity in many different ways due to their overall objectives, experience and life situation. Human activities are fluctuating. They may vary by individual and also over time within one's context of life. In order for a crossmedia system to be able to support activities throughout this fluctuation, a certain degree of functional modularity needs to be maintained. Functional modularity means that regardless of the intended synergistic structure of the system, its components or at least its primary or master 
component should allow for it to be operated to some degree also independently. For example, in addition to supporting exercise, the heart rate monitor provides limited support for planning and follow-up as well. For more advanced support, users may use the web service, but when they do not have time for it or their threshold to using it is too high, they will be adequately supported by the heart rate monitor as well. The degree of functional modularity is determined by the mutual dependencies among the components and can be applied when the components' hierarchical position allows for it.

Crossmedia systems may provide optimal means for supporting many levels of a human activity by providing layered support through their various components. Users who carry out their activities at a lower level of elaborateness, do well using only a part of the system. Whereas more advanced levels of an activity may be supported by providing more extensive functionalities through additional media. A high level of synergistic specificity, i.e., making it impossible to use one component without the use others, may intimidate users that only need a certain level of support and perceive the threshold to use the whole system as too overwhelming. However, having the opportunity to extend the use of tools as the nature of one's activity evolves over time, towards more elaborate or advanced, is one of the benefits of these kinds of systems. The roles of the system components need to be identified with respect to the primary activity, as well as with respect to each other. The hierarchical structure of the system is determined by the roles of, and dependencies among its components. Identifying this hierarchical structure provides a framework by which distribution of functionality may be applied. Functional modularity helps to maintain a moderate degree of flexibility that enables the system to be used on several different levels of an activity and by different users.

Even though some of the implications presented above may be applicable outside the scope of this particular study, they are still based on an analysis of a specific system and activity domain. Further research is needed in order to form a broader understanding of how crossmedia systems could be structured in various application environments, such as learning, and shopping. It would also be interesting to investigate, whether there are distinct compositional patterns that support some situated types better than others. Despite being designed to enable synergistic use practices, crossmedia also needs to actively support them and for that, the development of interaction techniques that lower users' thresholds to adopt synergistic use practices is needed. An interesting direction for research and development would be systems or IT environments that combine context aware and ubiquitous computing with the concept of crossmedia to support everyday life through the activities that constitute it.

\section{Conclusion}

This paper reports a three-month-long qualitative field study with 12 participants using a crossmedia fitness system, comprising a heart rate monitor and a web service, to support their training. The aim was to investigate how the composition of the crossmedia system influenced the ways in which it and its components would be utilized. It was found that the quality of users' primary motivation to exercise, elaborateness of their activities, internalization or externalization of their actions as well 
as their perceived threshold to using distinct IT artifacts determined the utilization of the system and each of its components. Structural and compositional aspects of the system influenced its ability to support different ways of training.

Implications are focused on the design of crossmedia systems in terms of their structure and composition. A system structure that corresponds with the target human activity and incorporates a hierarchical structure of roles among its components may be able to provide a clear system image and appropriately layered support for the primary activity. However, a certain degree of modularity needs to be maintained in order to support the different levels of elaborateness that an activity may be carried out on. Further research is needed to form a broader understanding of how crossmedia may support other situated types or human activities as well as to identify different compositional patterns that may be applied to crossmedia.

Acknowledgements. I wish to thank the RichWeb project, National Technology Agency of Finland (TEKES), Graduate School of Software Systems and Engineering (SoSE), Oulun Yliopiston Tukisäätiö, and Polar Electro Oy for funding parts of this research. I am grateful to Marja Harjumaa for her contribution to the study. I also wish to thank Kari Kuutti and Tanja Kotro for commenting the manuscript and the Stanford HCI Group, Harri Oinas-Kukkonen, Esa Juusola, Tanja Kalliojärvi, Sirpa Hope, and Petteri Siekkinen for their support and Svante Kärkkäinen and SATS Oulu fitness centers for cooperation.

\section{References}

1. Aneja, S., Lim, Y.-K.: Designing for Totality of Mobile and Non-Mobile Interaction: A Case Study. In: Ext. Abstracts CHI 2007, pp. 1685-1690 (2007)

2. Beyer, H., Holzblatt, K.: Contextual Design: Defining Customer-Centered Systems. Morgan Kaufmann Publishers/ Academic Press, San Francisco (1998)

3. Boumans, J.: Cross Media: E-content report 8. Published in a series of E-Content Reports by ACTeN (2004), http: / / www . acten. net

4. Edwards, K.W., Newman, M.W., Sedivy, J.Z.: Building the Ubiquitous Computing User Experience. In: Ext. Abstracts CHI 2001, pp. 501-502. ACM Press, New York (2001)

5. Feiner, S.K.: Environment Management for Hybrid User Interfaces. IEEE Personal Communications, 50-53 (October 2000)

6. Filgueiras, L., Correa, D., Oliveira Neto, J., Facis, R.: X-gov planning: how to apply cross media to government services. In: ICDS 2008, pp. 140-145. IEEE, Los Alamitos (2008)

7. Johanson, B., Fox, A., Winograd, T.: Experiences with Ubiquitous Computing Rooms. IEEE Pervasive Computing, 67-74 (2002)

8. Kuutti, K.: Activity Theory as a Potential Framework for Human-Computer Interaction Research. In: Nardi, B. (ed.) Context and Consciousness. Activity Theory and HumanComputer Interaction. The MIT Press, Cambridge (1996)

9. Laukkanen, R., Virtanen, P.: Heart Rate Monitors: State of the Art. Journal of Sports Sciences 16(3) (suppl. 1), 3-7 (1998)

10. McCullough, M.: Digital Ground. Architecture, Pervasive Computing, and Environmental Knowing. The MIT Press, Cambridge (2005)

11. Nardi, B.: Context and Consciousness. Activity Theory and Human-Computer Interaction. The MIT Press, Cambridge (1996) 
12. Newman, M., Sedivy, J., Neuwirth, C., Edwards, W.K., Hong, J., Izadi, S., Marcelo, K., Smith, T.: Designing for serendipity: supporting end-user configuration of ubiquitous computing environments. In: Proc. of the 4th conference on Designing Interactive Systems, London, pp. 147-156. ACM, New York (2002)

13. Nilsson, M.: Advancements and Trends in Medical Case-Based Reasoning: An Overview of Systems and System Development. In: Proc. International FLAIRS Conference, Miami, USA, pp. 178-183 (2004)

14. Oulasvirta, A., Sumari, L.: Mobile Kits and Laptop Trays: Managing Multiple Devices in Mobile Information Work. In: Proc. CHI 2007, pp. 1127-1136. ACM Press, New York (2007)

15. Patton, M.Q.: Qualitative evaluation and research methods, 2nd edn. Sage Publications, Newbury Park (1990)

16. Schilling, M.A.: Towards a general modular systems theory and its application to interfirm product modularity. Academy of Management Review 25, 312-334; Reprinted in Garud, R., Langlois, D., Kumaraswamy, A. (eds) Managing in the Modular Age: Architectures, Networks and Organizations. Blackwell Publishers, Oxford (2000)

17. Seaman, J.: Adopting a Grounded Theory Approach to Cultural-Historical Research: Conflicting Methodologies or Complementary Methods? International Journal of Qualitative Methods 7(1) (2008)

18. Simon, H.: The Architecture of Complexity. Proceedings of the American Philosophical Society 106, 467-782 (1962)

19. Steen-Johnsen, K.: Globalized Fitness in the Norwegian Context: The Perfect Meets the Popular. International Review for the Sociology of Sport 42(3), 343-362 (2007)

20. Wiberg, C., Jegers, K., Bodén, J.: Cross Media Interaction Design. In: Presented at the workshop HCI and New Media Arts: Methodology and Evaluation at CHI 2007 (2007) 